\title{
Alterations in Defense Enzymes by Azoxystrobin and Chaetoglobosin Biomolecules in Chilli
}

\author{
V.M. Srinivasan* and A.S. Krishnamoorthy \\ Department of Plant Pathology, Tamil Nadu Agricultural University, \\ Coimbatore 641 003, India \\ *Corresponding author
}

\begin{abstract}
A B S T R A C T
The activity of defense enzymes viz., peroxidase, polyphenol oxidase, catalase and

\section{Keywords}

Azoxystrobin, Chaetoglobosin, Defense enzymes, Chilli

Article Info

Accepted:

10 February 2019

Available Online:

10 March 2019 superoxide dismutase up on treatment with azoxystrobin and chaetoglobosin was studied in this experiment. The chilli plants inoculated with C.capsici showed various levels of peroxidase activity with respect to the treatments. The combined application of azoxystrobin with chaetoglobosin at 0.2 per cent concentration showed the highest level of peroxidase activity (1.130). The next highest level (0.872) was observed in azoxystrobin with tebuconazole combination. In the case of chilli plants inoculated with L. taurica, the maximum peroxidase activity was recorded in the same combination (0.817). The highest polyphenol oxidase activity in chilli plants inoculated with C.capsici recorded in azoxystrobin and chaetoglobosin combination $(0.883)$. The next best increase $(0.721)$ of PPO was noted in combined application of azoxystrobin with tebuconazole at 0.2 per cent concentration. The same trend was noticed when chilli plant inoculated with L. taurica. In the case of catalase and superoxide dismutase, azoxystrobin and chaetoglobosin combination, followed by azoxystrobin (Willowood) and tebuconazole combination showed the highest level of activity in chilli plants inoculated with $C$. capsici and L.taurica.
\end{abstract}

\section{Introduction}

The activity of defense enzymes viz., peroxidase (PO), polyphenol oxidase (PPO), phenylalanine ammonia lyase (PAL), $\beta-1,3$ glucanase, chitinase, catalase and defense inducing chemicals (total phenols) was found to be increased in azoxystrobin and $P$. fluorescens treated chilli plants (Amrita et al., 2016). Increased expression of specific isoforms of PO and PPO was observed due to Induced Systemic Resistance (ISR) induction
(Zahidi et al., 2018). Peroxidase polymorphism could be used as a biochemical marker related to different levels of field resistance (Lebeda et al., 1999). Peroxidases also participate in synthesis of phenolic compounds and in the building of intermolecular bonds during the organization of the cell wall at the sites of infection by the pathogens (Repka and Slovakova, 1994). Many studies have shown that PPO is induced in response to mechanical wounding; fungal and bacterial infection; treatment with 
signaling molecules such as jasmonic acid / methyl jasmonate (MeJA); system in and salicylic acid (Yadav et al., 2017). Sundravadana (2008) reported that, azoxystrobin had efficiently activated the defense enzymes viz., PO, PPO, and PAL which are increased the lignin content in $P$. grisea inoculated rice seedlings. Systemic induction of PPO in response to wounding and pathogen infection might provide an additional line of defense to protect the plants against further attack by pathogen and insects (Thipyapong et al., 1995).

Application of salicylic acid on bluegrass plants increased the activity of catalase and super oxide dismutase (Mckersie et al., 1996). Babitha (2002) reported the higher SOD activity in resistant pearl millet seedlings than the susceptible seedlings upon inoculation with Sclerospora graminicola. The fungicides such as ketoconazole, propiconazole and azoxystrobin increased the production of antioxidant enzymes viz., superoxide dismutase, catalase, and peroxidases in plants (Abdul et al., 2008; Gonias et al., 2008).

\section{Materials and Methods}

Induction of defense related enzymes in chilli up on treatment with azoxystrobin and chaetoglobosin was assayed by using the methodologies given below. In all the experiments, tebuconazole was included for comparison purpose.

\section{Assay of defense related enzymes}

The fungicide azoxystrobin at 0.2 per cent concentration were compared with 0.2 per cent of chaetoglobosin and tebuconazole for the induction of defense related enzymes. Chilli plants sprayed with above treatments and where inoculated with Colletotrichum capsici, Leveilulla taurica. The leaf samples were collected at $0,1,3,5$,
7, $9 \mathrm{~d}$ after inoculation of the pathogen and used for enzyme assay.

One g of chilli leaf sample was homogenized with one $\mathrm{ml}$ of $0.1 \mathrm{M}$ Sodium phosphate buffer $(\mathrm{pH} 7.0)$ at $4{ }^{0} \mathrm{C}$. The homogenate was centrifuged for $20 \mathrm{~min}$ at $10000 \mathrm{rpm}$. The supernatant was used as enzyme extract for assaying of Peroxidase (PO) and Poly Phenol Oxidase (PPO). For Catalase and Super oxide Dismutase (SOD) the sample was extracted in $5 \mathrm{ml}$ of $0.05 \mathrm{M}$ sodium acetate buffer $(\mathrm{pH}$ 5.0). The homogenate was centrifuged at $20,000 \mathrm{rpm}$ for $10 \mathrm{~min}$ at $4^{\circ} \mathrm{C}$ and the supernatant was used as enzyme source.

\section{Assay of peroxidase (PO)}

Assay of PO activity was carried out as per the procedure described by Hammerschmidt et al., (1995). The reaction mixture consisted of $2.5 \mathrm{ml}$ of the mixture containing $0.25 \%$ (v/v) guaiacol in $0.01 \mathrm{M}$ sodium phosphate buffer, pH 6.0 and $0.1 \mathrm{M}$ hydrogen peroxide. Enzyme extract $(0.1 \mathrm{ml})$ was added to initiate the reaction, which was followed calorimetrically at $470 \mathrm{~nm}$. Crude enzyme preparations were diluted to give changes in absorbance at $470 \mathrm{~nm}$ of 0.1 to 0.2 absorbance units / min. The boiled enzyme was used as blank. Activity was expressed as the increase in absorbance at $420 \mathrm{~nm} \mathrm{~min}^{-1}$ $\mathrm{mg}^{-1}$ of protein.

\section{Assay of polyphenoloxidase (PPO)}

The polyphenoloxidase activity was determined as per the procedure given by Mayer et al., (1965). The reaction mixture consisted of $1.5 \mathrm{ml}$ of $0.1 \mathrm{M}$ sodium phosphate buffer (pH 6.5) and $200 \mu$ of the enzyme extract. To start the reaction, $200 \mu \mathrm{l}$ of $0.01 \mathrm{M}$ catechol was added and the activity was expressed as change in absorbance at 495 $\mathrm{min}^{-1} \mathrm{mg}^{-1}$ of protein. 


\section{Assay of catalase (CAT)}

CAT activity was assayed spectrophotometrically as described by Chaparro-Giraldo et al., (2000) using $3 \mathrm{ml}$ assay mixture containing $100 \mathrm{mM}$ potassium phosphate buffer ( $\mathrm{pH}$ 7.5) and $2.5 \mathrm{mM} \mathrm{H}_{2} \mathrm{O}_{2}$ prepared immediately before use and $100 \mu \mathrm{l}$ enzyme extract. The activity was measured by monitoring the degradation of $\mathrm{H}_{2} \mathrm{O}_{2}$ using UV-Visible Spectrophotometer (Varian Cary 50) at $240 \mathrm{~nm}$ over $1 \mathrm{~min}$, against a plant extract-free blank. The decrease in $\mathrm{H}_{2} \mathrm{O}_{2}$ was followed as the decline in optical density at $240 \mathrm{~nm}$, activity was calculated using the extinction coefficient $\left(\varepsilon_{240 \mathrm{~nm}}=40 \mathrm{mM}^{-1} \mathrm{~cm}^{-1}\right)$ for $\mathrm{H}_{2} \mathrm{O}_{2}$ and expressed in $\mu \mathrm{mol} \mathrm{min}^{-1} \mathrm{mg}^{-1}$ of sample.

\section{Assay of superoxide dismutase (SOD)}

The enzyme extract was prepared by homogenizing $1 \mathrm{~g}$ of chilli tissue in $2 \mathrm{ml}$ of $0.2 \mathrm{M}$ citrate phosphate buffer $(\mathrm{pH} 6.5)$ at $4^{\circ} \mathrm{C}$. The homogenate was centrifuged at $15,000 \mathrm{~g}$ at $4^{\circ} \mathrm{C}$ for $30 \mathrm{~min}$. The supernatant served as enzyme source and SOD activity (EC 1.15.1.1) was determined as its ability to inhibit the photochemical reduction of NBT. The assay mixture $(3 \mathrm{ml})$ contained $50 \mathrm{mM}$ sodium phosphate buffer ( $\mathrm{pH} 7.8$ ), $13 \mathrm{mM}$ methionine, $75 \mu \mathrm{M}$ NBT, $2 \mu \mathrm{M}$ riboflavin. $0.1 \mathrm{mM}$ EDTA and $100 \mu \mathrm{l}$ of the enzyme extract and the riboflavin was added at the end. Tubes were shaken and placed under a $40-\mathrm{W}$ fluorescent lamp at $25^{\circ} \mathrm{C}$.

The reaction was initiated and terminated by turning the light on and off respectively. The absorbance at $560 \mathrm{~nm}$ was measured against identical non-illuminated in parallel to the sample tubes for blank. Each extract was subtracted from the blank and mathematical difference was then divided by blank and multiplied by 100 to obtain the percentage inhibition of NBT photo-reduction. The SOD activity was expressed in SOD units $\mathrm{mg}^{-1}$ tissue (50\% NBT inhibition = 1 unit) (ElMoshaty et al., 1993).

\section{Results and Discussion}

\section{Changes in peroxidase (PO)}

The chilli plants inoculated with C.capsici showed various levels of peroxidase activity with respect to the treatments. The combined application of azoxystrobin with chaetoglobosin at 0.2 per cent concentration showed the highest level of peroxidase activity (1.130). The next highest level (0.872) was observed in azoxystrobin with tebuconazole combination. The lowest peroxidase activity (0.157) was observed in uninoculated control.

In the case of chilli plants inoculated with $L$. taurica, the maximum peroxidase activity was recorded in combined application of azoxystrobin (Willowood) with chaetoglobosin (0.817). The next highest (0.725) peroxidase activity observed in individual application of azoxystrobin; azoxystrobin and tebuconazole combination (0.702) also showed significant increase of peroxidase activity than the inoculated (0.231) and uninoculated (0.158) control (Table 1 and 2; Fig. 1 and 2).

\section{Changes in polyphenoloxidase (PPO)}

The experimental results revealed that the activity of polyphenol oxidase was progressively increased in all the treatments when compared to both inoculated $(0.183)$ and uninoculated (0.152) control. Among the different treatments, combination of azoxystrobin with chaetoglobosin recorded the highest polyphenol oxidase activity in chilli plants inoculated with $C$.capsici $(0.883)$. The next best increase (0.721) of PPO was noted in combined application of 
azoxystrobin with tebuconazole at 0.2 per cent concentration. The same trend was noticed when chilli plant inoculated with $L$. taurica. The highest PPO (1.016) activity was noticed when both biomolecules were combined. The next best increase of PPO activity was noticed in combination of azoxystrobin with tebuconazole (0.936) which is onpar with tebuconazole and chaetoglobosin (0.901) combination and individual application of azoxystrobin (0.917) and chaetoglobosin (0.889) (Table 2 and 3; Fig. 3 and 4).

\section{Changes in catalase (CAT)}

Azoxystrobin (Willowood) and chaetoglobosin combination (0.942), followed by azoxystrobin (Willowood) and tebuconazole combination (0.761) showed the highest level of catalase activity in chilli plants inoculated with $C$. capsici. The lowest catalase activity was noticed in uninoculated (0.364) control.In the case of chilli plants inoculated with L.taurica, the same combination showed the highest (0.895) level of catalase activity (Table 5 and 6; Fig. 5 and $6)$.

\section{Changes in superoxide dismutase (SOD)}

The combined application of azoxystrobin (Willowood) with chaetoglobosin at 0.2 per cent concentration on chilli showed the highest SOD activity against C.capsici and L.taurica (9.32 and 8.84). Which is followed by, the combination of azoxystrobin with tebuconazole showed the second maximum increase of SOD activity against both the pathogens (9.00 and 8.06). The minimum SOD activity was recorded in uninoculated control (Table 7 and 8; Fig. 7 and 8).

Table.1 Effect of azoxystrobin, chaetoglobosin and tebuconazole on peroxidase activity in chilli plants inoculated with $C$. capsici

\begin{tabular}{|c|c|c|c|c|c|c|}
\hline \multirow[t]{2}{*}{ Treatment } & \multicolumn{6}{|c|}{$\begin{array}{c}\text { Absorbance at } 420 \eta \min ^{-1} \mathrm{~g}^{-1} \text { at different } \\
\text { intervals (d) }\end{array}$} \\
\hline & 0 & 1 & 3 & 5 & 7 & 9 \\
\hline Azoxystrobin $0.2 \%$ & $0.364^{\mathrm{a}}$ & $0.542^{\mathrm{a}}$ & $0.613^{b}$ & $0.736^{\mathrm{cd}}$ & $0.802^{\text {cd }}$ & $0.721^{\text {cd }}$ \\
\hline Chaetoglobosin $0.2 \%$ & $0.329^{b}$ & $0.531^{\mathrm{a}}$ & $0.604^{\mathrm{b}}$ & $0.765^{\mathrm{cd}}$ & $0.739^{\mathrm{de}}$ & $0.718^{\text {cd }}$ \\
\hline Tebuconazole $0.2 \%$ & $0.290^{c}$ & $0.484^{\mathrm{b}}$ & $0.620^{\mathrm{b}}$ & $0.701^{\mathrm{d}}$ & $0.728^{\mathrm{e}}$ & $0.692^{d}$ \\
\hline $\begin{array}{l}\text { Azoxystrobin } 0.2 \%+\text { Chaetoglobosin } \\
0.2 \%\end{array}$ & $0.336^{\mathrm{b}}$ & $0.542^{\mathrm{a}}$ & $0.802^{\mathrm{a}}$ & $1.158^{\mathrm{a}}$ & $1.134^{\mathrm{a}}$ & $1.130^{\mathrm{a}}$ \\
\hline $\begin{array}{l}\text { Tebuconazol } 0.2 \% \text { + Chaetoglobosin } \\
0.2 \%\end{array}$ & $0.278^{\mathrm{cd}}$ & $0.322^{\text {cd }}$ & $0.532^{\mathrm{c}}$ & $0.792 b^{c}$ & $0.840^{\mathrm{c}}$ & $0.762^{c}$ \\
\hline $\begin{array}{l}\text { Azoxystrobin } 0.2 \%+\text { Tebuconazol } \\
0.2 \%\end{array}$ & $0.298^{c}$ & $0.336^{c}$ & $0.571^{\mathrm{bc}}$ & $0.847^{\mathrm{b}}$ & $0.918^{b}$ & $0.872^{b}$ \\
\hline Inoculated Control & $0.263^{d}$ & $0.287^{\mathrm{d}}$ & $0.349^{\mathrm{d}}$ & $0.368^{\mathrm{e}}$ & $0.305^{\mathrm{f}}$ & $0.276^{\mathrm{e}}$ \\
\hline Un inoculated control & $0.143^{e}$ & $0.175^{\mathrm{e}}$ & $0.218^{\mathrm{e}}$ & $0.226^{\mathrm{f}}$ & $0.191^{g}$ & $0.157^{f}$ \\
\hline
\end{tabular}

Mean of three replications

In a column, means followed by same letter are not significantly different at the 5 per cent level by DMRT 
Table.2 Effect of azoxystrobin, chaetoglobosin and tebuconazole on peroxidase activity in chilli plants inoculated with $L$. taurica

\begin{tabular}{|c|c|c|c|c|c|c|}
\hline \multirow[t]{2}{*}{ Treatment } & \multicolumn{6}{|c|}{ Absorbance at $420 \eta \min _{\text {(d) }}^{-1} \mathrm{~g}^{-1}$ at different intervals } \\
\hline & $\mathbf{0}$ & 1 & 3 & 5 & 7 & 9 \\
\hline Azoxystrobin $0.2 \%$ & $0.298^{\mathrm{a}}$ & $0.486^{\mathrm{b}}$ & $0.627^{b}$ & $0.741^{b c}$ & $0.739^{b}$ & $0.725^{b}$ \\
\hline Chaetoglobosin $0.2 \%$ & $0.315^{\mathrm{a}}$ & $0.493^{b}$ & $0.574^{\text {cd }}$ & $0.726^{\mathrm{c}}$ & $0.696^{b}$ & $0.684^{b}$ \\
\hline Tebuconazole $0.2 \%$ & $0.267^{b}$ & $0.407^{\mathrm{c}}$ & $0.562^{\mathrm{d}}$ & $0.698^{\mathrm{c}}$ & $0.679^{\mathrm{b}}$ & $0.622^{\mathrm{c}}$ \\
\hline $\begin{array}{l}\text { Azoxystrobin } 0.2 \%+ \\
\text { Chaetoglobosin } 0.2 \%\end{array}$ & $0.306^{\mathrm{a}}$ & $0.592^{\mathrm{a}}$ & $0.708^{\mathrm{a}}$ & $0.958^{\mathrm{a}}$ & $0.925^{\mathrm{a}}$ & $0.817^{\mathrm{a}}$ \\
\hline $\begin{array}{l}\text { Tebuconazol } 0.2 \%+ \\
\text { Chaetoglobosin } 0.2 \%\end{array}$ & $0.218^{\mathrm{c}}$ & $0.322^{\mathrm{d}}$ & $0.532^{\mathrm{d}}$ & $0.792^{\mathrm{b}}$ & $0.710^{\mathrm{b}}$ & $0.693^{b}$ \\
\hline $\begin{array}{l}\text { Azoxystrobin } 0.2 \%+\text { Tebuconazol } \\
0.2 \%\end{array}$ & $0.290^{\mathrm{a}}$ & $0.484^{\mathrm{b}}$ & $0.620^{\mathrm{bc}}$ & $0.736^{\mathrm{bc}}$ & $0.714^{\mathrm{b}}$ & $0.702^{b}$ \\
\hline Inoculated Control & $0.261^{\mathrm{b}}$ & $0.284^{\mathrm{e}}$ & $0.316^{\mathrm{e}}$ & $0.286^{\mathrm{d}}$ & $0.273^{c}$ & $0.231^{\mathrm{d}}$ \\
\hline Un inoculated control & $0.136^{d}$ & $0.159^{f}$ & $0.207^{\mathrm{f}}$ & $0.226^{\mathrm{e}}$ & $0.197^{d}$ & $0.158^{\mathrm{e}}$ \\
\hline
\end{tabular}

Mean of three replications

In a column, means followed by same letter are not significantly different at the 5 per cent level by DMRT

Table.3 Effect of azoxystrobin, chaetoglobosin and tebuconazole on polyphenol oxidase (PPO) activity in chilli plants inoculated with $C$. capsici

\begin{tabular}{|c|c|c|c|c|c|c|}
\hline \multirow[t]{2}{*}{ Treatment } & \multicolumn{6}{|c|}{ Absorbance at $495 \eta \mathrm{m} \mathrm{min} \mathrm{m}^{-1} \mathrm{~g}^{-1}$ at different intervals (d) } \\
\hline & $\mathbf{0}$ & 1 & 3 & 5 & 7 & 9 \\
\hline Azoxystrobin $0.2 \%$ & $0.283^{\mathrm{abc}}$ & $0.394^{\mathrm{d}}$ & $0.551^{\mathrm{d}}$ & $0.856^{\mathrm{b}}$ & $0.748^{c}$ & $0.673 b$ \\
\hline Chaetoglobosin $0.2 \%$ & $0.294^{\mathrm{a}}$ & $0.425^{\mathrm{c}}$ & $0.676^{\mathrm{b}}$ & $0.896^{\mathrm{b}}$ & $0.751^{\mathrm{c}}$ & $0.607^{c}$ \\
\hline Tebuconazole $0.2 \%$ & $0.260^{\text {bcd }}$ & $0.367^{\mathrm{e}}$ & $0.644^{\mathrm{bc}}$ & $0.740^{\mathrm{c}}$ & $0.680^{\mathrm{d}}$ & $0.413^{e}$ \\
\hline $\begin{array}{l}\text { Azoxystrobin } 0.2 \%+\text { Chaetoglobosin } \\
0.2 \%\end{array}$ & $0.288^{\mathrm{a}}$ & $0.462^{b}$ & $0.794^{\mathrm{a}}$ & $0.995^{\mathrm{a}}$ & $0.927^{\mathrm{a}}$ & $0.883^{\mathrm{a}}$ \\
\hline $\begin{array}{l}\text { Tebuconazol } 0.2 \%+\text { Chaetoglobosin } \\
0.2 \%\end{array}$ & $0.253^{\mathrm{d}}$ & $0.346^{\mathrm{f}}$ & $0.594^{\mathrm{cd}}$ & $0.829^{\mathrm{b}}$ & $0.795^{\mathrm{b}}$ & $0.501^{\mathrm{d}}$ \\
\hline $\begin{array}{l}\text { Azoxystrobin } 0.2 \%+\text { Tebuconazol } 0.2 \\
\%\end{array}$ & $0.259^{\mathrm{cd}}$ & $0.536^{\mathrm{a}}$ & $0.678^{\mathrm{b}}$ & $0.890^{\mathrm{b}}$ & $0.743^{\mathrm{c}}$ & $0.721^{b}$ \\
\hline Inoculated Control & $0.284^{\mathrm{ab}}$ & $0.289^{\mathrm{g}}$ & $0.297^{\mathrm{e}}$ & $0.212^{\mathrm{d}}$ & $0.206^{\mathrm{e}}$ & $0.183^{f}$ \\
\hline Un inoculated control & $0.200^{e}$ & $0.230^{h}$ & $0.260^{\mathrm{e}}$ & $0.200^{d}$ & $0.190^{\mathrm{e}}$ & $0.152^{f}$ \\
\hline
\end{tabular}

Mean of three replications

In a column, means followed by same letter are not significantly different at the 5 per centlevelby DMRT 
Table.4 Effect of azoxystrobin, chaetoglobosin and tebuconazole on polyphenol oxidase (PPO) activity in chilli plants inoculated with $L$. taurica

\begin{tabular}{|c|c|c|c|c|c|c|}
\hline \multirow[t]{2}{*}{ Treatment } & \multicolumn{6}{|c|}{ Absorbance at $495 \eta \mathrm{m} \mathrm{min} \mathrm{m}^{-1} \mathrm{~g}^{-1}$ at different intervals (d) } \\
\hline & $\mathbf{0}$ & 1 & 3 & 5 & 7 & 9 \\
\hline Azoxystrobin $0.2 \%$ & $0.313^{\mathrm{c}}$ & $0.629^{\mathrm{bc}}$ & $0.847^{\mathrm{a}}$ & $0.971^{\mathrm{bc}}$ & $0.944^{\mathrm{b}}$ & $0.917^{b}$ \\
\hline Chaetoglobosin $0.2 \%$ & $0.394^{\mathrm{b}}$ & $0.643^{\mathrm{b}}$ & $0.796^{\mathrm{ab}}$ & $0.923^{\mathrm{bc}}$ & $0.901^{\mathrm{b}}$ & $0.889^{b}$ \\
\hline Tebuconazole $0.2 \%$ & $0.278^{\mathrm{e}}$ & $0.464^{\mathrm{de}}$ & $0.685^{\mathrm{d}}$ & $0.892^{\mathrm{c}}$ & $0.879^{\mathrm{b}}$ & $0.853^{\mathrm{c}}$ \\
\hline $\begin{array}{l}\text { Azoxystrobin } 0.2 \% \text { + Chaetoglobosin } \\
0.2 \%\end{array}$ & $0.337^{\mathrm{c}}$ & $0.698^{\mathrm{a}}$ & $0.847^{\mathrm{a}}$ & $1.274^{\mathrm{a}}$ & $1.112^{\mathrm{a}}$ & $1.016^{\mathrm{a}}$ \\
\hline $\begin{array}{l}\text { Tebuconazol } 0.2 \%+\text { Chaetoglobosin } \\
0.2 \%\end{array}$ & $0.311^{\mathrm{cd}}$ & $0.583^{\mathrm{c}}$ & $0.729^{\mathrm{cd}}$ & $0.984^{\mathrm{b}}$ & $0.916^{\mathrm{b}}$ & $0.901^{b}$ \\
\hline $\begin{array}{l}\text { Azoxystrobin } 0.2 \%+\text { Tebuconazol } 0.2 \\
\%\end{array}$ & $0.434^{\mathrm{a}}$ & $0.496^{\mathrm{d}}$ & $0.752^{\mathrm{bc}}$ & $0.956^{\mathrm{bc}}$ & $0.944^{b}$ & $0.936^{b}$ \\
\hline Inoculated Control & $0.282^{\text {de }}$ & $0.437^{\mathrm{e}}$ & $0.579^{\mathrm{e}}$ & $0.703^{d}$ & $0.688^{\mathrm{c}}$ & $0.659^{d}$ \\
\hline Un inoculated control & $0.264^{e}$ & $0.379^{\mathrm{f}}$ & $0.485^{f}$ & $0.691^{d}$ & $0.653^{c}$ & $0.614^{d}$ \\
\hline
\end{tabular}

Mean of three replications

In a column, means followed by same letter are not significantly different at the 5 per cent level by DMRT

Table.5 Effect of azoxystrobin, chaetoglobosin and tebuconazole on catalase activity in chilli plants inoculated with $C$. capsici

\begin{tabular}{|c|c|c|c|c|c|c|}
\hline \multirow[t]{2}{*}{ Treatment } & \multicolumn{6}{|c|}{$\begin{array}{c}\text { changes in absorbance at } 240 \mathrm{~nm} \text { min- }{ }^{1} \mathrm{~g}^{-1} \text { at different } \\
\text { intervals (d) }\end{array}$} \\
\hline & $\mathbf{0}$ & 1 & 3 & 5 & 7 & 9 \\
\hline Azoxystrobin 0.2\% & $0.448^{\mathrm{ab}}$ & $0.653^{\mathrm{a}}$ & $0.774^{\mathrm{ab}}$ & $0.859^{\text {cd }}$ & $0.792^{\mathrm{bc}}$ & $0.718^{b}$ \\
\hline Chaetoglobosin $0.2 \%$ & $0.397^{\mathrm{c}}$ & $0.510 \mathrm{c}$ & $0.760^{\mathrm{ab}}$ & $0.844^{\mathrm{cd}}$ & $0.738^{\mathrm{c}}$ & $0.701^{\text {bc }}$ \\
\hline Tebuconazole $0.2 \%$ & $0.402^{\mathrm{c}}$ & $0.629^{\mathrm{ab}}$ & $0.731^{\mathrm{bc}}$ & $0.824^{\mathrm{d}}$ & $0.769^{\mathrm{bc}}$ & $0.652^{\text {cd }}$ \\
\hline $\begin{array}{l}\text { Azoxystrobin } 0.2 \%+\text { Chaetoglobosin } \\
0.2 \%\end{array}$ & $0.472^{\mathrm{a}}$ & $0.643^{\mathrm{a}}$ & $0.814^{\mathrm{a}}$ & $1.106^{\mathrm{a}}$ & $0.983^{\mathrm{a}}$ & $0.942^{\mathrm{a}}$ \\
\hline $\begin{array}{l}\text { Tebuconazol } 0.2 \% \text { + Chaetoglobosin } \\
0.2 \%\end{array}$ & $0.324^{\mathrm{d}}$ & $0.590^{\mathrm{b}}$ & $0.684^{\mathrm{c}}$ & $0.912^{\mathrm{bc}}$ & $0.812^{\mathrm{b}}$ & $0.624^{d}$ \\
\hline $\begin{array}{l}\text { Azoxystrobin } 0.2 \%+\text { Tebuconazol } 0.2 \\
\%\end{array}$ & $0.423^{\mathrm{bc}}$ & $0.612^{\mathrm{ab}}$ & $0.763^{\mathrm{ab}}$ & $0.960^{\mathrm{b}}$ & $0.818^{\mathrm{b}}$ & $0.761^{b}$ \\
\hline Inoculated Control & $0.337^{\mathrm{d}}$ & $0.339^{\mathrm{e}}$ & $0.427^{\mathrm{e}}$ & $0.518^{\mathrm{e}}$ & $0.483^{\mathrm{d}}$ & $0.497^{\mathrm{e}}$ \\
\hline Un inoculated control & $0.323^{d}$ & $0.394^{d}$ & $0.516^{d}$ & $0.430^{f}$ & $0.356^{\mathrm{e}}$ & $0.364^{f}$ \\
\hline
\end{tabular}

Mean of three replications

In a column, means followed by same letter are not significantly different at the 5 per cent level by DMRT 
Table.6 Effect of azoxystrobin, chaetoglobosin and tebuconazole on catalase activity in chilli plants inoculated with $L$. taurica

\begin{tabular}{|c|c|c|c|c|c|c|}
\hline \multirow[t]{2}{*}{ Treatment } & \multicolumn{6}{|c|}{$\begin{array}{l}\text { changes in absorbance at } 240 \mathrm{~nm} \text { min- }{ }^{1} \mathrm{~g}^{1}{ }^{1} \text { at } \\
\text { different intervals (d) }\end{array}$} \\
\hline & $\mathbf{0}$ & 1 & 3 & 5 & 7 & 9 \\
\hline Azoxystrobin $0.2 \%$ & $0.323^{\mathrm{c}}$ & $0.475^{\mathrm{cd}}$ & $0.691^{b}$ & $0.862^{b c}$ & $0.799^{b}$ & $0.747^{b}$ \\
\hline Chaetoglobosin $0.2 \%$ & $0.307^{\mathrm{cd}}$ & $0.618^{\mathrm{a}}$ & $0.688^{b}$ & $0.749^{d}$ & $0.701^{\mathrm{c}}$ & $0.695^{\mathrm{bc}}$ \\
\hline Tebuconazole $0.2 \%$ & $0.397 \mathrm{a}$ & $0.443^{\mathrm{d}}$ & $0.617^{\mathrm{c}}$ & $0.798^{\text {cd }}$ & $0.593^{\mathrm{d}}$ & $0.574^{d}$ \\
\hline $\begin{array}{l}\text { Azoxystrobin } 0.2 \%+\text { Chaetoglobosin } \\
0.2 \%\end{array}$ & $0.301^{\mathrm{d}}$ & $0.558^{\mathrm{b}}$ & $0.827^{\mathrm{a}}$ & $0.974^{\mathrm{a}}$ & $0.912^{\mathrm{a}}$ & $0.895^{\mathrm{a}}$ \\
\hline $\begin{array}{l}\text { Tebuconazol } 0.2 \%+\text { Chaetoglobosin } \\
0.2 \%\end{array}$ & $0.385^{\mathrm{a}}$ & $0.476^{\mathrm{cd}}$ & $0.589^{\mathrm{cd}}$ & $0.798^{\text {cd }}$ & $0.683^{c}$ & $0.641^{c}$ \\
\hline $\begin{array}{l}\text { Azoxystrobin } 0.2 \%+\text { Tebuconazol } \\
0.2 \%\end{array}$ & $0.324^{\mathrm{c}}$ & $0.497^{\mathrm{c}}$ & $0.686^{\mathrm{b}}$ & $0.907^{\mathrm{ab}}$ & $0.896^{\mathrm{a}}$ & $0.874^{\mathrm{a}}$ \\
\hline Inoculated Control & $0.360^{\mathrm{b}}$ & $0.389^{\mathrm{e}}$ & $0.571^{\mathrm{cd}}$ & $0.594^{\mathrm{f}}$ & $0.567^{\mathrm{de}}$ & $0.496^{\mathrm{e}}$ \\
\hline Un inoculated control & $0.301^{d}$ & $0.469^{\mathrm{cd}}$ & $0.543^{d}$ & $0.677^{\mathrm{e}}$ & $0.522^{\mathrm{e}}$ & $0.470^{\mathrm{e}}$ \\
\hline
\end{tabular}

Mean of three replications

In a column, means followed by same letter are not significantly different at the 5 per cent level by DMRT

Table.7 Effect of azoxystrobin, chaetoglobosin and tebuconazole on superoxide dismutase (SOD) activity in chilli plants inoculated with $C$. capsici

\begin{tabular}{|c|c|c|c|c|c|c|}
\hline \multirow[t]{2}{*}{ Treatment } & \multicolumn{6}{|c|}{$\begin{array}{c}\text { Unit / min / } \mathrm{g} \text { of sample at } 560 \mathrm{~nm} \text { in different } \\
\text { intervals (d) }\end{array}$} \\
\hline & $\mathbf{0}$ & 1 & 3 & 5 & 7 & 9 \\
\hline Azoxystrobin $0.2 \%$ & $3.651^{\mathrm{ab}}$ & $4.89^{\mathrm{a}}$ & $6.12^{c}$ & $8.87^{\mathrm{a}}$ & $9.34^{b}$ & $9.00^{\mathrm{a}}$ \\
\hline Chaetoglobosin $0.2 \%$ & $3.293^{\mathrm{cd}}$ & $3.57^{\mathrm{c}}$ & $5.84^{\mathrm{c}}$ & $7.94^{\mathrm{b}}$ & $7.18^{c}$ & $6.62^{c}$ \\
\hline Tebuconazole $0.2 \%$ & $3.562^{b c}$ & $4.21^{b}$ & $4.56^{\mathrm{d}}$ & $5.22^{d}$ & $5.42^{d}$ & $4.96^{d}$ \\
\hline $\begin{array}{l}\text { Azoxystrobin } 0.2 \%+ \\
\text { Chaetoglobosin } 0.2 \%\end{array}$ & $3.368^{\mathrm{bc}}$ & $5.24^{\mathrm{a}}$ & $7.49^{\mathrm{a}}$ & $9.21^{\mathrm{a}}$ & $10.33^{\mathrm{a}}$ & $9.32^{\mathrm{a}}$ \\
\hline $\begin{array}{l}\text { Tebuconazol } 0.2 \%+ \\
\text { Chaetoglobosin } 0.2 \%\end{array}$ & $3.016^{\mathrm{de}}$ & $4.45^{b}$ & $5.73^{c}$ & $6.49^{c}$ & $5.91^{d}$ & $5.48^{d}$ \\
\hline $\begin{array}{l}\text { Azoxystrobin } 0.2 \%+\text { Tebuconazol } \\
0.2 \%\end{array}$ & $3.941^{\mathrm{a}}$ & $4.29^{b}$ & $6.73^{b}$ & $8.71^{\mathrm{a}}$ & $7.78^{c}$ & $7.32^{b}$ \\
\hline Inoculated Control & $3.393^{b c}$ & $3.74^{\mathrm{c}}$ & $3.84^{\mathrm{e}}$ & $3.96^{\mathrm{e}}$ & $3.94^{\mathrm{e}}$ & $3.55^{\mathrm{e}}$ \\
\hline Un inoculated control & $2.980^{\mathrm{e}}$ & $2.67^{d}$ & $3.24^{f}$ & $2.34^{\mathrm{f}}$ & $2.68^{f}$ & $2.12^{\mathrm{f}}$ \\
\hline
\end{tabular}

Mean of three replications

In a column, means followed by same letter are not significantly different at the 5 per cent level by DMRT 
Table.8 Effect of azoxystrobin, chaetoglobosin and tebuconazole on superoxide dismutase (SOD) activity in chilli plants inoculated with L. taurica

\begin{tabular}{|l|c|c|c|c|c|c|}
\hline \multirow{2}{*}{ Treatment } & \multicolumn{5}{|c|}{ Unit / min / g of sample at 560 nm in different } \\
intervals (d)
\end{tabular}

Mean of three replications

In a column, means followed by same letter are not significantly different at the 5 per cent level by DMRT

Fig.1

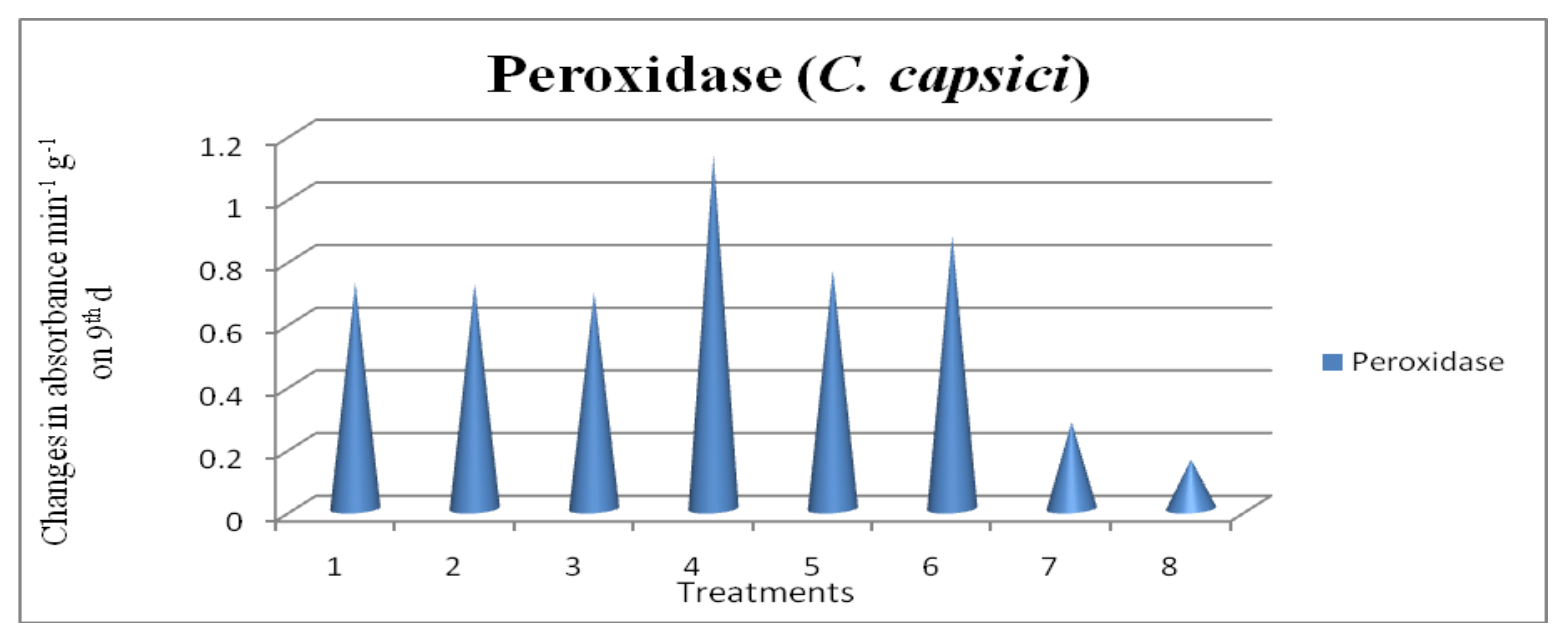

Fig.2

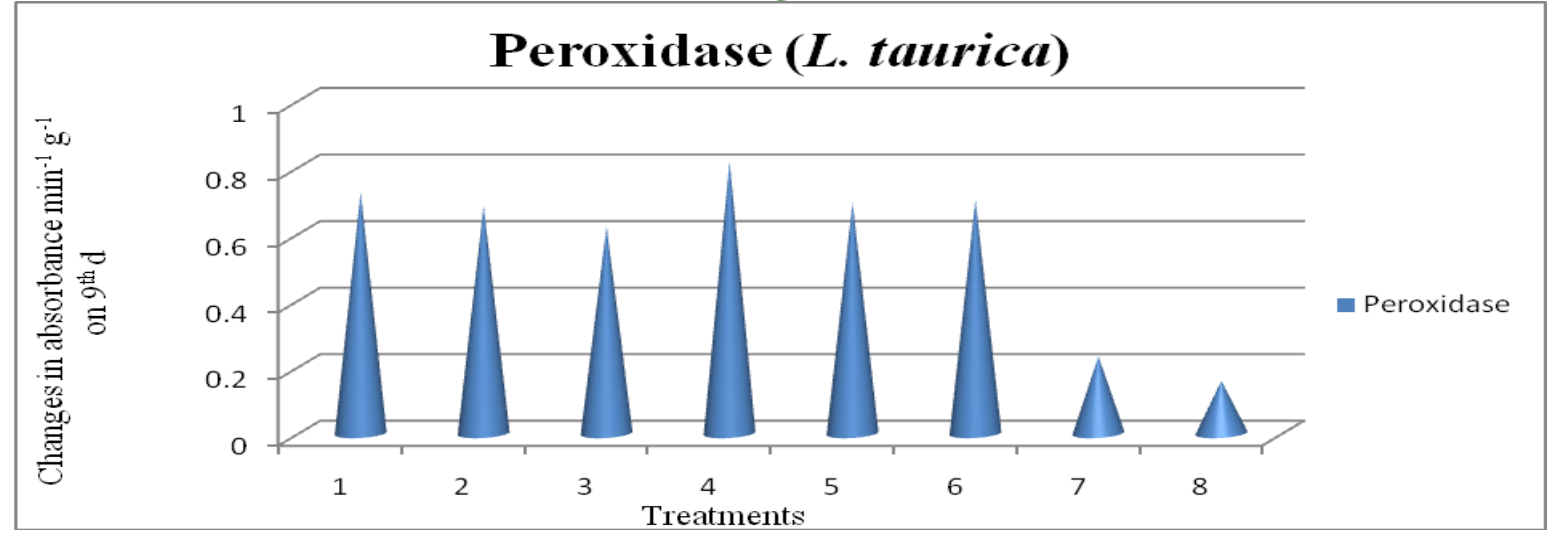


Fig.3

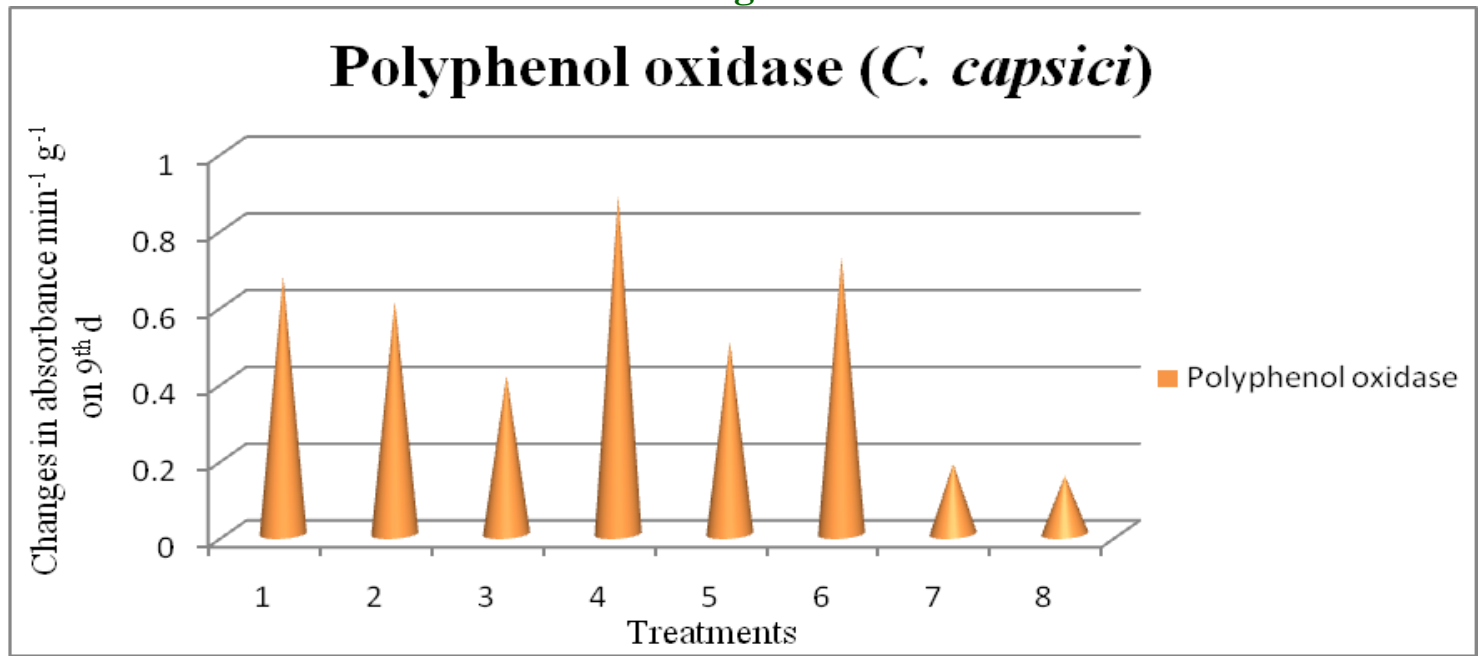

Fig.4

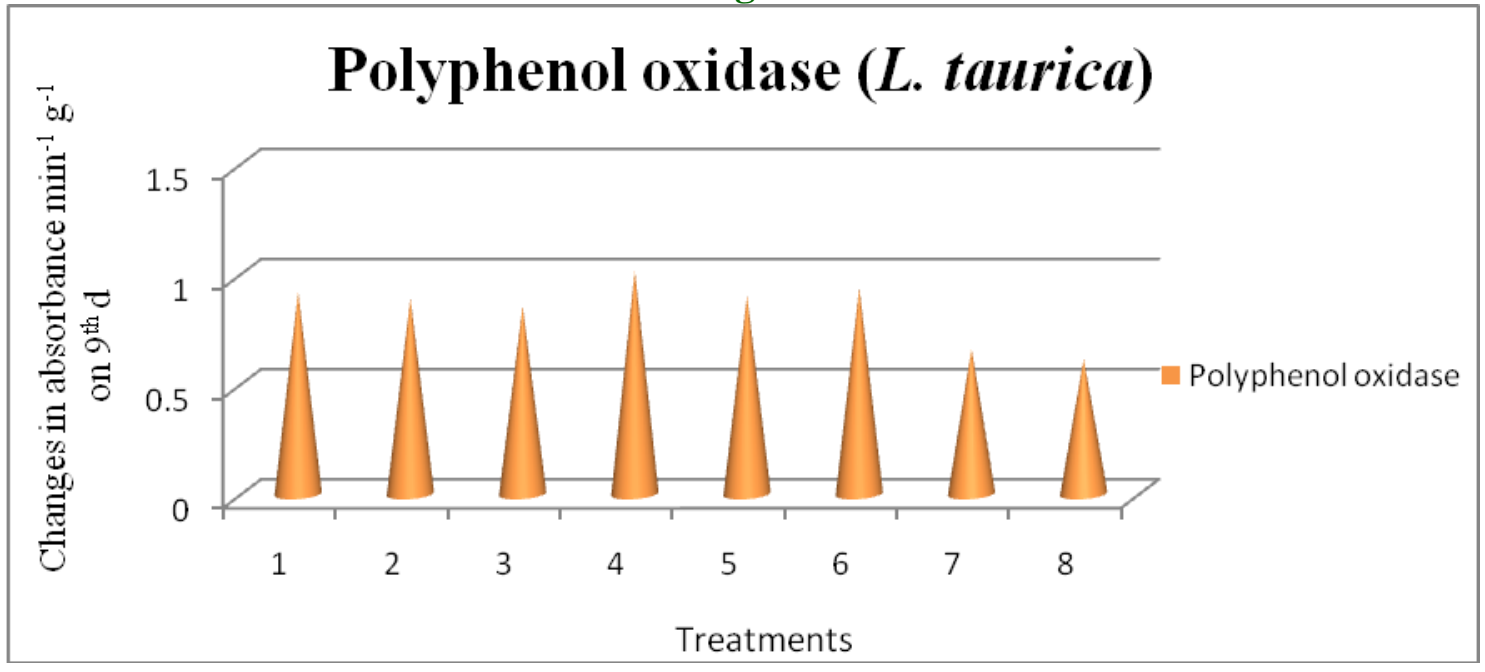

Fig.5

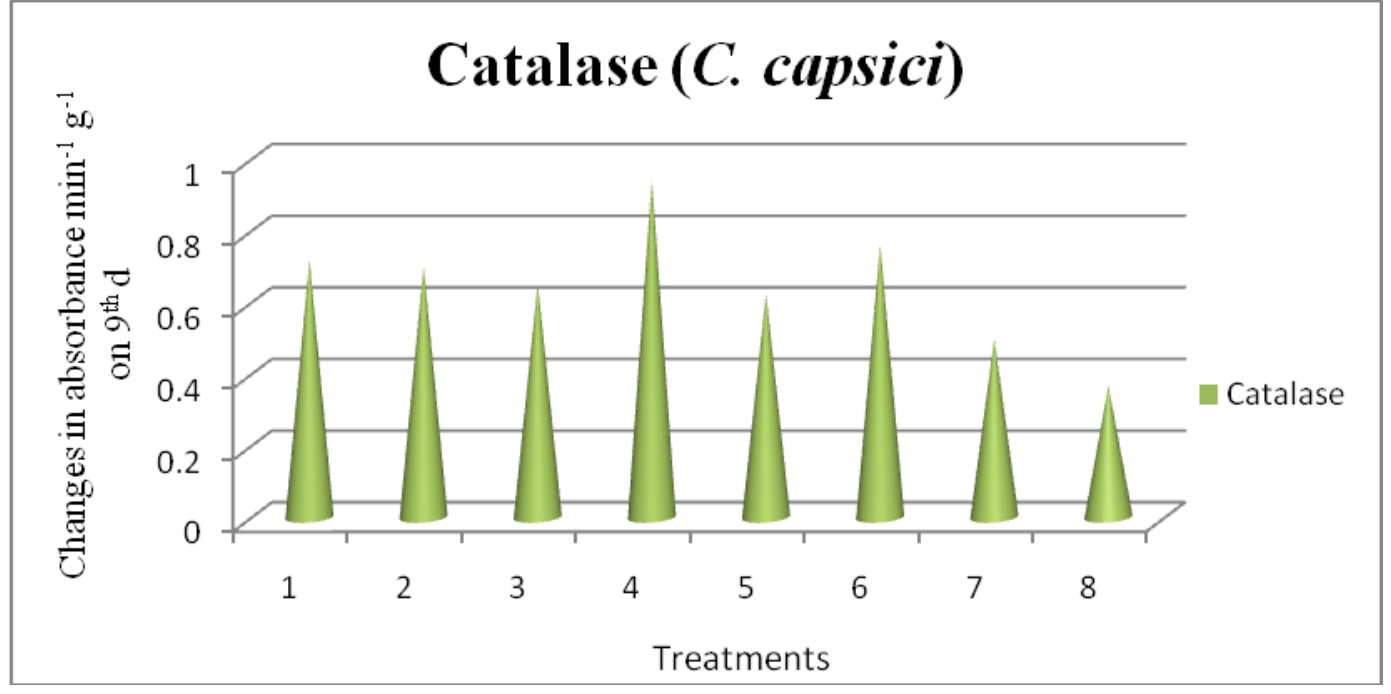


Fig.6

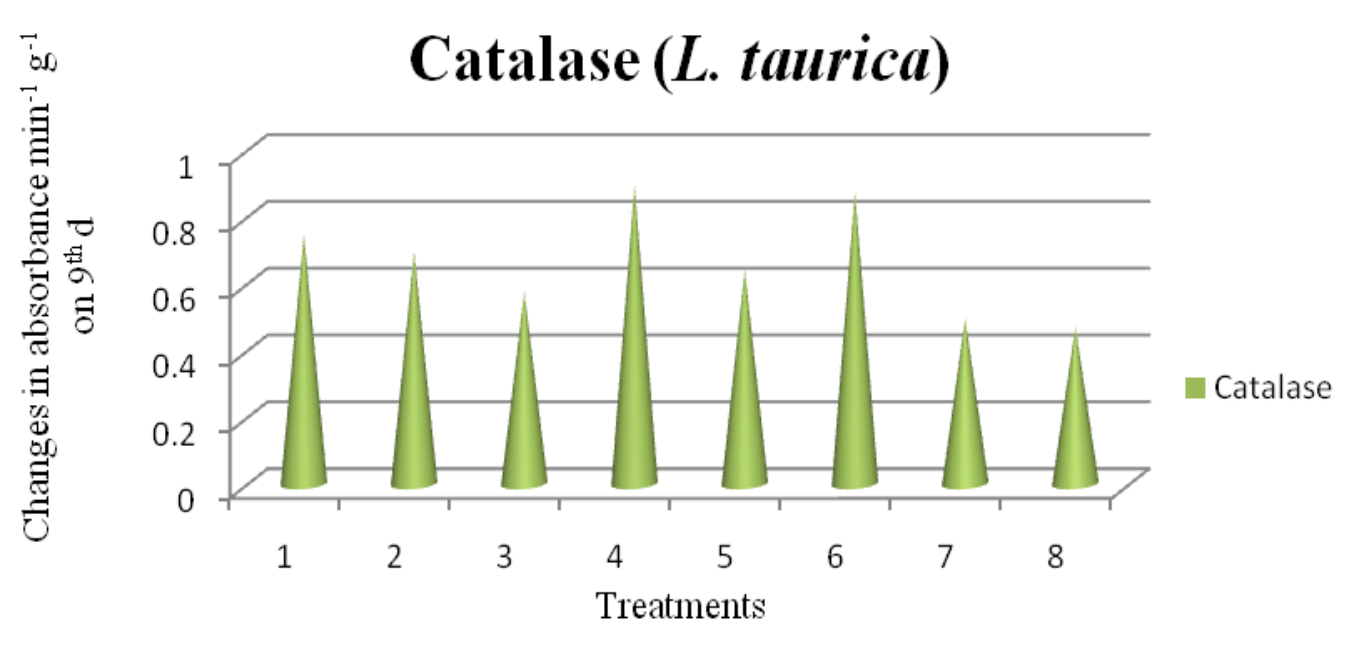

Fig.7

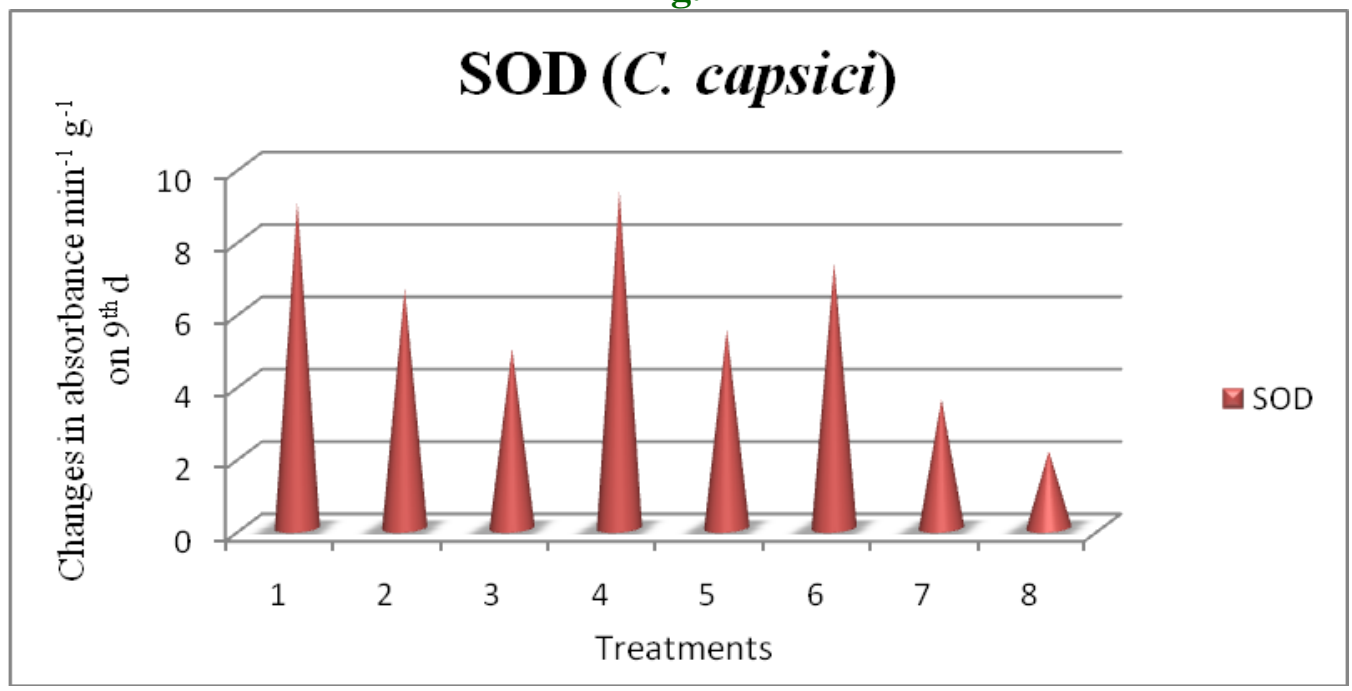

Fig.8

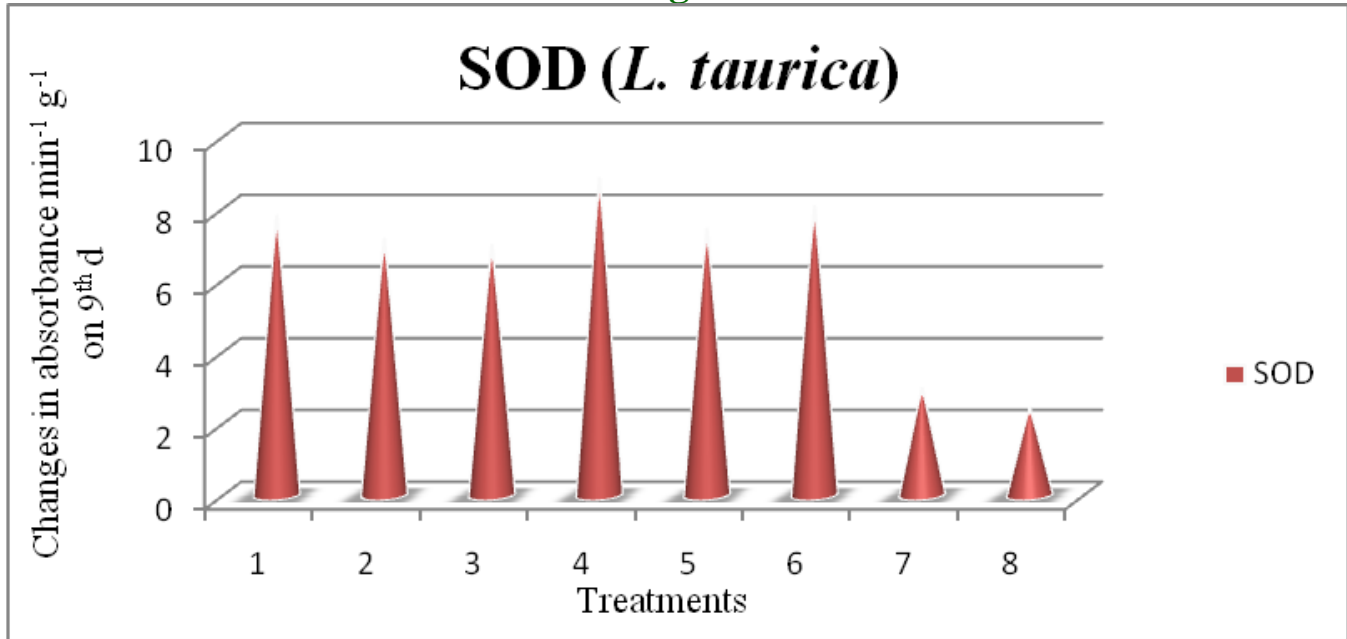


Exposing plants to abiotic or biotic stresses lead to improved resistance to subsequent pathogen attack both locally and systemically (Walter et al., 2005). Applying fungicides on plants was also found to induce the resistance against the pathogens. For example, pyraclostrobin (strobilurin class fungicide) enhanced resistance of tobacco plants by activation of pathogenesis related protein (PR 1) against Tobacco Mosaic Virus and Pseudomonas syringae pv tabaci (Herms et al., 2002). The defense enzymes such as superoxide dismutase, catalase and ascorbate peroxidase activities increased after the application of metalaxyl on Solanum nigrum (Alexandra et al., 2013). In the present study also, triggering of defense related enzymes viz., peroxidase (PO), polyphenol oxidase (PPO), catalase and super oxide dismutase (SOD) was recorded in chilli plant upon spraying with azoxystrobin, chaetoglobosin and other fungicides. The individual application of fungicides showed lesser increase in defense enzymes as compared to combination treatments. Among the combinations, azoxystrobin with chaetoglobosin showed the maximum induction of defense enzymes in chilli. Similar reports have already been made by Nuchadomrong et al., 2004. They reported that the activity of the defense enzymes such as peroxidase (PO), polyphenol oxidase (PPO), phenyl alanine ammonia lyase (PAL) and chitinase increased in the azoxystrobin treated cucumber plants.

The bioactive compounds, trichotoxin A50 extracted from Trichoderma harzianum PC01 and chaetoglobosin $\mathrm{C}$ extracted from Chaetomium globosum have also been reported to elicit resistance or immunity in plants by inducing oxidative burst in plant cells (Reihana et al., 2018). Hence, apart from various modes of action, inducing resistance in plants is an additional advantage for azoxystrobin as well as for chaetoglobosin application, which will be most helpful in managing the plant diseases effectively.

\section{References}

Abdul, J.C., Gopi, R., Manivannan, P., Gomathinayagam, M., Murali, P. V. and Panneerselvam, R. 2008. Soil applied propiconazole alleviates the impact of salinity on Catharanthus roseus by improving antioxidant status. Pestic. Biochem. Phys., 90:135-139.

Alexendra, S., Singh, U.P., Sarma, B.K., Nishimura, R., Kobayashi, K., Akira, O., Volker, Z., Barbel, S.B. and Aust, H.J . 2013. Differential growth response of A1 and A2 mating types of Phytophthora infestans on Rye A and V-8 Juice Agar Media supplemented with rhizome powder of Cyperus rotundus. Mycobiology, 29(3): 164-169.

Amrita, S., Richa, R., Vijay, K.G and Harikesh, B.S .2016. Chilli anthracnose : epidemiology and management. Front Microbiol. 7: 1527.

Babitha, M.P., Bhat, S.G., Prakash, H.S. and. Shetty, H.S. 2002. Differential induction of super oxide dismutase in downy mildew resistance and susceptible genotypes of pearl millet. Plant Pathology, 51: 480-486.

Chaparro-Giraldo, A., Barata, R.M., Chabregas, S.M., Azevedo, R.A. and Silva-Filho, M.C. 2000. Soybean leghemoglobin targeted to potato chloroplasts influences growth and development of transgenic plants. Plant Cell Rep., 19: 961-965.

El-Moshaty, B., Pike, S.M., Novacky, A.J., Sehgal, O.P.1993. Lipid peroxidation and superoxide production in cowpea (Vigna unguiculata) leaves infected with tobacco ring spot virus or 
southern bean mosaic virus. Physiol. Mol. Plant Pathol. 43:109-119.

Gonias, E. D., Oosterhuis, D. M. and Bibi, A. C. 2008. Physiological response of cotton to the insecticide imidacloprid under high - temperature stress. $J$. Plant Growth Regul., 27: 77-82.

Herms, S., Seehaus, K., Koehle, H., Conrath, U. 2002. A strobilurin fungicide enhances the resistance of tobacco against tobacco mosaic virus and Pseudomonas syringae pv tabaci. Plant Physiol., 130: 120-27.

Lebeda, A. 1999..Pseudoperonospora cubensis on Cucumis spp. and Cucurbita spp. - resistance breeding aspects. Acta Hort., 492:363-370.

Mayer, A.M., Harel, E. and Shaul, R.B. 1965. Assay of catechol oxidase a critical comparison of methods. Phytochemistry, 5: 783-789.

McKersie, B.D., Bowley, S.R., Harjanto, E., Leprice, O.1996. Water-deficit tolerance and field performance of transgenic alfalfa over expressing superoxide dismutase. Plant Physiology, 111: 1321-1326.

Nuchadomrong, S., Buthongsri, P., Kano Kmedhalkul, S. and Soytong, K. 2004. Trichotoxin A50: Induction of an oxidative defence mechanism in tomato and its cellular site of action. In proceedings 30th congress on Science and Technology of Thailand, 19-21 Oct. Impact Muang Thong Thani, Bangkok, Thailand.

Reihana, R., Mohd, Y.R., Ismail, S.I., Martini, M.Y., Gous, M., and Magaji, U. 2018. Breeding for
Anthracnose Disease Resistance in Chili: Progress and Prospects. Int.J.Mol.Sci. 19(10): 3122.

Repka,V and Slovonosa. 1994. Structure, expression and synthesis induction of proteins related to pathogenic processes in plant cell. Biol. Listy., 58: 81-100.

Sundravadana, S., Alice, D., Kuttalam, S. and Samiyappan, R. 2007. Azoxystrobin induces lignification-related enzymes and phenolics in rice (Oryza sativa L.) against blast pathogen (Pyricularia grisea). J. Plant Interactions, 2: 219 224.

Thipyapong, P., Hunt, M.D., Steffens, J.C. 1995. Antisense down regulation of polyphenol oxidase results in enhanced disease susceptibility. Planta, 220:105-117.

Walter, D., Walsh, D., Newton, A. and. Lyon, G.2005. Induced resistance for plant disease control: maximizing the efficacy of resistance elicitors. Phytopathology, 95:1368-1373.

Yadav, A.L., Ghasolia, R.P., Choudhary, S and Yadav, V.K. 2017. Exploitation of fungicides and plant extracts for ecofriendly management of chilli fruit rot disease. Int.J.Chem. Std. 5(4):1632-1634.

Zahidi, R., Lekchiri, A., Berrichi, A., Mimouni, M. and Halouani, E,L. 2018. Extraction and comparison of two new peroxidases from leaves and roots of Brassica oleraceae var. ramosa J. Mater. Environ. Sci. 9(5): 1398-1404.

\section{How to cite this article:}

Srinivasan, V.M. and Krishnamoorthy, A.S. 2019. Alterations in Defense Enzymes by Azoxystrobin and Chaetoglobosin Biomolecules in Chilli. Int.J.Curr.Microbiol.App.Sci. 8(03): 1191-1202. doi: https://doi.org/10.20546/ijcmas.2019.803.141 